\title{
TWO-GRID WEAK GALERKIN METHOD FOR SEMILINEAR ELLIPTIC DIFFERENTIAL EQUATIONS
}

\author{
luoping chen ${ }^{1}$, fanyun $\mathrm{wu}^{1}$, and guoyan zeng ${ }^{1}$ \\ ${ }^{1}$ Southwest Jiaotong University
}

January 14, 2022

\begin{abstract}
In this paper, we investigate a two-grid weak Galerkin method for semilinear elliptic differential equations. The method mainly contains two steps. First, we solve the semi-linear elliptic equation on the coarse mesh with mesh size H, then, we use the coarse mesh solution as a initial guess to linearize the semilinear equation on the fine mesh, i.e., on the fine mesh (with mesh size $\$ \mathrm{~h} \$$ ), we only need to solve a linearized system. Theoretical analysis shows that when the exact solution u has sufficient regularity and $\$ \mathrm{~h}=\mathrm{H}^{\wedge} 2 \$$, the two-grid weak Galerkin method achieves the same convergence accuracy as weak Galerkin method. Several examples are given to verify the theoretical results.
\end{abstract}

\section{Hosted file}

two-grid weak galerkin method.pdf available at https://authorea.com/users/455522/articles/ 552811-two-grid-weak-galerkin-method-for-semilinear-elliptic-differential-equations 